\title{
Cross-tier interference management schemes in cognitive heterogeneous networks
}

\author{
loannis Tzelatis ${ }^{*}$ and Kostas Berberidis
}

\begin{abstract}
To satisfy the ever-increasing data rate and service coverage demands, wireless communication networks evolve into heterogeneous networks (HetNets), where low-cost small base stations are embedded in conventional macrocells. Intercell interference emerges as the key capacity-limiting factor in such dense networks, restricting the reusability of spectral resources. Therefore, advanced interference mitigation techniques relying on multi-cell cooperation have attracted significant attention from the wireless industry and academia. This paper discusses interference management schemes for multi-tiered spectrum access in next-generation HetNets. A novel scheme based on full system cognition and base station cooperation is proposed as an enabler for high-capacity HetNets. In addition, practical implementation and operational challenges are investigated.
\end{abstract}

Keywords: Interference management; Heterogeneous networks; Spectrum sharing; Cognitive radio

\section{Introduction}

One of the major challenges for next-generation wireless communication systems is to enhance the system capacity and cope with the ever-increasing mobile traffic demands. Due to the scarcity of available spectrum, the spectral efficiency needs to be improved in order to meet the demands. Today's deployed networks are mostly based on macrocells and are characterized as homogeneous, in the sense that all base stations (BS) belong to the same type and power class. While macrocells are the basis of a mobile communication network, heterogeneous deployments have been investigated over the past few years as a means to provide very high data rates for in-home communications or within an enterprise environment.

In such enterprise tiered deployments, low-cost small base stations, such as femtocells [1], are embedded in conventional macrocells. Femtocells are short-ranged (10 to $30 \mathrm{~m}$ ) low-power access points developed to provide cost-effective high-capacity services within a small coverage area. The cell splitting aims at reducing the distance between transmission and reception points to increase the achievable data rates. Moreover, a frequency reuse factor of 1 is applied, meaning that the deployed base stations share the full available spectrum to improve the spectral

\footnotetext{
*Correspondence: tzelatis@ceid.upatras.gr

Department of Computer Engineering and Informatics, University of Patras, Patras 26500, Greece
}

efficiency and achieve high peak throughput. However, due to the close proximity of the transmission points, heterogeneous networks (HetNets) suffer from harsh interference conditions. Intercell interference emerges as the key limiting factor, restricting the reusability of the spectral resources. Two types of interference occur within a multi-tier network architecture, co-tier and cross-tier interferences. The first occurs among network elements of the same type (e.g., between neighboring femtocells), and the latter among network elements that belong to different tiers (e.g., between macrocell and femtocell).

Intercell interference management techniques have recently attracted growing interest $[2,3]$. The conventional non-cooperative approach is to treat intercell interference as noise at the receiver. This may be characterized as passive treatment. Another more proactive solution to manage intercell interference is to apply interferenceaware multi-cell coordination at the base stations [4]. Such coordination methods require backhaul links to facilitate the base station cooperation via information exchange. The degree of cooperation defines a trade-off between the performance gains and the resulting signalling overhead. Some representative coordination-based methods are discussed in next paragraph.

According to the technique proposed in [5], the frequency spectrum is divided into two parts, a macrocell dedicated part and a macrocell-femtocell shared spectrum

\section{照 Springer}

(c) 2014 Tzelatis and Berberidis; licensee Springer. This is an Open Access article distributed under the terms of the Creative Commons Attribution License (http://creativecommons.org/licenses/by/4.0), which permits unrestricted use, distribution, and reproduction in any medium, provided the original work is properly credited. 
part. Macrocell users that pose a threat to nearby femtocells are assigned resources from the dedicated spectrum part. This scheme, however, becomes inefficient if the number of macrocell users near femtocells increases. In [6] and [7], clustering of femtocells based on their geographical locations is investigated. The authors in [8] propose that cross-tier interference can be mitigated if a femtocell can avoid using the macrocell resources that belong to its nearby macrocell users through efficient spectrum sensing. Dynamic partitioning of resources into several sub-bands with fractional frequency reuse (FFR) is proposed in [9-11]. A cognitive radio approach based on distributed spectrum sensing is presented in [12]. Yet, the main disadvantage of all these approaches is the fact that each BS can use only parts of the available spectrum at a given time, thus limiting the overall spectral efficiency. Other methods entail power control and focus on reducing the transmission power of femtocells [12-14].

Cognitive radio (CR) technology has been extensively investigated over the past few years with several research areas. One of the main topics is how to achieve efficient spectrum sharing. CR technology addresses the problem of spectrum utilization and refers to systems that are aware of their environment. Typically, a CR network consists of legacy service subscribers and cognitive users. The legacy subscribers are called licensed or primary users and have priority to access the frequency spectrum. The cognitive users are called unlicensed or secondary users and are allowed to reuse the same radio resources, as long as they do not create significant performance degradation to the primary users. This can be achieved in different ways [15]. In interweave CR networks, the secondary users monitor the spectrum and access it only when they sense an unused slot [16]. In underlay CR networks, the secondary users are allowed to utilize the same frequency spectrum as the primary users in a carefully controlled manner by limiting the resulting interference under predefined thresholds [17].

Spectrum sharing via dynamic spectrum access (DSA) allows a frequency reuse factor of 1 , thus offering increased spectral efficiency. Therefore, DSA has been investigated in the literature (e.g., [18-20]) as a means to optimize HetNets and manage intercell interference. More specifically, in [18,19], dynamic spectrum access for cognitive femtocell networks is investigated, where the femtocell users are considered as secondary users with lower priority compared to the macrocell (primary) users. In [20], opportunistic cooperation for cognitive femtocell networks is proposed, where the femtocell nodes either assist the macrocell transmissions as relays or defer their transmissions to protect the macrocell users from interference. Nevertheless, in most realistic cases, the femtocell users are regular subscribers with high data rate demands, and down-prioritizing them over macrocell users poses obvious rate limitations. These limitations are crucial, given that femtocells can be considered as a viable network solution only if they can ensure high capacity, which in turn justifies the additional deployment costs.

In this paper, we discuss the potential benefits of cognition for managing cross-tier intercell interference in HetNets by deviating from the conventional CR model. Similar to $[5,21]$ co-tier interference is considered as noise and the focus is on cross-tier interference. In particular, we consider and propose full system cognition and multi-cell cooperation, where all BSs are cognitive nodes. This means that the BSs have more knowledge about their environment (e.g., channel state information and network architecture). The focus is on a two-tier cellular system, where multiple femtocell base stations (FBS) are deployed within the coverage of a single macrocell base station (MBS).

Different interference management techniques are considered in conjunction with resource allocation. We develop and propose a novel scheme which exploits the network architecture and additional BS information to achieve efficient interference management and improve the system capacity. Joint precoding with multi-cell cooperation is investigated to this end. Joint precoding has been extensively investigated in the literature [4]. However, the novelty of this work lies in the way the precoder is computed and applied in conjunction with resource allocation. Moreover, we propose a practical adaptation of the theoretical scheme which considers realistic assumptions regarding the channel state information at the BSs. Finally, we show how such a scheme can be utilized by cognitive HetNets leading to efficient spectrum usage. The proposed scheme is compared to two reference schemes, one being a non-cognitive solution, as defined by 3GPP for LTE-Advanced [22], and the other one being a conventional CR solution [17].

The remainder of this paper is organized as follows. In Section 2, we introduce the underlying system model. Section 3 presents the transmission schemes under consideration, while Section 4 provides extensive simulation results and the respective performance analysis. Practical implementation and operational challenges are also discussed. Finally, Section 5 concludes this article.

\section{System model}

One recent deployment trend, foreseen to be widely used for long-term evolution (LTE) advanced networks, consists of splitting the base station functionalities into a control unit, whose main task is scheduling, and several remote radio heads ( $R R H)$, responsible for all the radio frequency operations. The control unit is typically connected to the RRH via optical fiber, while the $\mathrm{RRH}$ is located close to the antennas. RRH deployments can therefore facilitate fast coordination between the 
transmission points. Here, we suggest a deployment as the one in Figure 1, where each FBS is realized as RRH, connected to the MBS via a fiber optic cable. It is assumed that the control unit is located in the MBS and that the backhaul link can offer sufficient capacity for real-time information exchange between the BSs.

Specifically, we consider a two-tier cellular radio network based on orthogonal frequency division multiplexing (OFDM) with $N_{\text {MBS }}$ transmit antennas per macrocell BS, $N_{\text {FBS }}$ transmit antennas per femtocell BS, and $M$ receive antennas per user terminal. Within a macrocell coverage area, there exist one MBS serving $P$ macro-users (MU) and $F$ FBSs serving $S$ femto-users (FU) each. We investigate cross-tier intercell interference management on the downlink, where the available physical resources are treated as a spectrum pool. FBSs are assigned nonoverlapping dedicated parts of equal bandwidth $B \mathrm{MHz}$ from the spectrum pool. This choice is not restrictive and each FBS could be assigned any part of the spectrum pool. The MBS can transmit over the whole spectrum to achieve high peak rates.

In such a system, the femtocells operating within the coverage of a single macrocell access different parts of the spectrum. Consequently, there is no co-tier interference among the femtocells of a given macrocell. Moreover, the interference between femtocells that operate within different macrocells is very low due to their low transmit power. Note also that femtocells can be deployed in such a way that neighboring femtocells from different macrocells do not access the same part of the spectrum. Thus, the only type of co-tier interference that is not negligible is the interference from neighboring macrocell base stations. In the existing literature, there have been several studies about the magnitude of macrocell intercell interference, and typical values range from -110 to $-65 \mathrm{dBm}$ $[23,24]$, which is relatively low compared to cross-tier interference. The focus of this work is on cross-tier interference between the MBS and the FBSs on the downlink channel. Therefore, any co-tier interference is considered as part of the noise, and other techniques like FFR and power control could be used to handle it.
For the system under consideration, the time axis is divided into transmit time intervals (TTIs) as a basic unit of time scheduling. Each dedicated spectrum part is divided into $K$ physical resource blocks (PRB), each comprised of a set of $L$ subsequent subcarriers. A PRB is regarded as the minimum resource unit in the frequency domain. Moreover, each BS transmits a single stream per served user.

The channel model used is depicted in Figure 2. We denote the downlink multiple-input multiple-output (MIMO) channel for the MBS-MU link by $\boldsymbol{H}$ and for the FBS-FU link by $\boldsymbol{G}$. The interfering links MBS-FU and FBSMU are denoted by $\tilde{\boldsymbol{H}}$ and $\tilde{\boldsymbol{G}}$, respectively. Let us also use indices $p$ and $s$ for the MU and FU, respectively, which are being served at a given TTI and PRB. Given a frequency reuse factor of 1 , each FBS can transmit synchronously with the MBS in a bandwidth of $B \mathrm{MHz}$. Assuming that precoding vectors $\boldsymbol{w}_{p} \in \mathbb{C}^{N_{\mathrm{MBS}}}, \boldsymbol{w}_{s} \in \mathbb{C}^{N_{\mathrm{FBS}}}$ are applied to the transmitted signal prior to transmission, the signal model for each pair of MU and FU can be formulated as

$$
\begin{aligned}
& \text { Macro-user : } \boldsymbol{y}_{p}=P_{p} \boldsymbol{H} \boldsymbol{w}_{p} x_{p}+P_{s} \tilde{\boldsymbol{G}} \boldsymbol{w}_{s} x_{s}+\boldsymbol{z}_{p} \\
& \text { Femto-user }: \boldsymbol{y}_{s}=P_{s} \boldsymbol{G} \boldsymbol{w}_{s} x_{s}+P_{p} \tilde{\boldsymbol{H}} \boldsymbol{w}_{p} x_{p}+\boldsymbol{z}_{s},
\end{aligned}
$$

where $x_{u}$ is the transmitted signal and $\boldsymbol{y}_{u} \in \mathbb{C}^{M}$ is the received vector signal for user $u$, with $u \in\{p, s\}$. The downlink channel matrices $\boldsymbol{H}, \tilde{\boldsymbol{H}} \in \mathbb{C}^{M x N_{M B S}}$ and $\boldsymbol{G}, \tilde{\boldsymbol{G}} \in \mathbb{C}^{M x N_{\mathrm{FBS}}}$ are considered as MIMO Rayleigh fading channels. This means that their individual channel elements are modelled as independent and identically distributed (i.i.d.) zero-mean complex Gaussian random variables with unit variance. $P_{u}^{2}$ is the transmit power for user $u$ and $z_{u}$ denotes the independent and identically distributed additive white Gaussian noise (AWGN), where $\boldsymbol{z}_{u} \sim C N\left(\mathbf{0}, N_{0} \mathbf{I}\right)$.

For the analysis and comparison of different interference management schemes, we assume in this article that full channel knowledge is available at the transmitter sides. Theoretically, this can be achieved by extensive

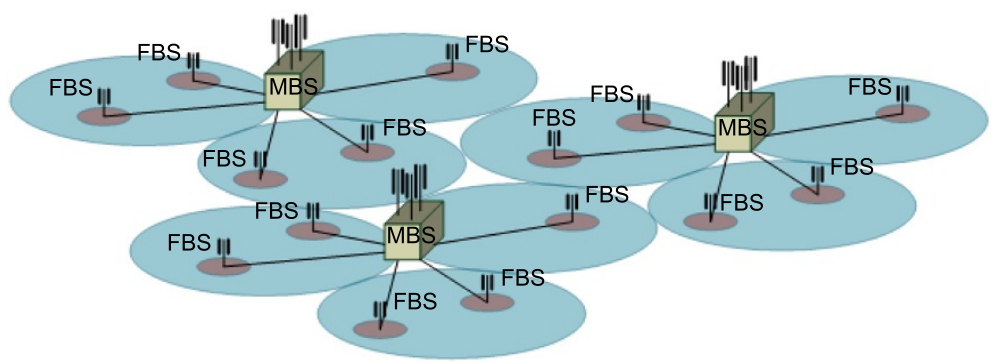

Figure 1 Heterogeneous network deployment with remote radio heads. 


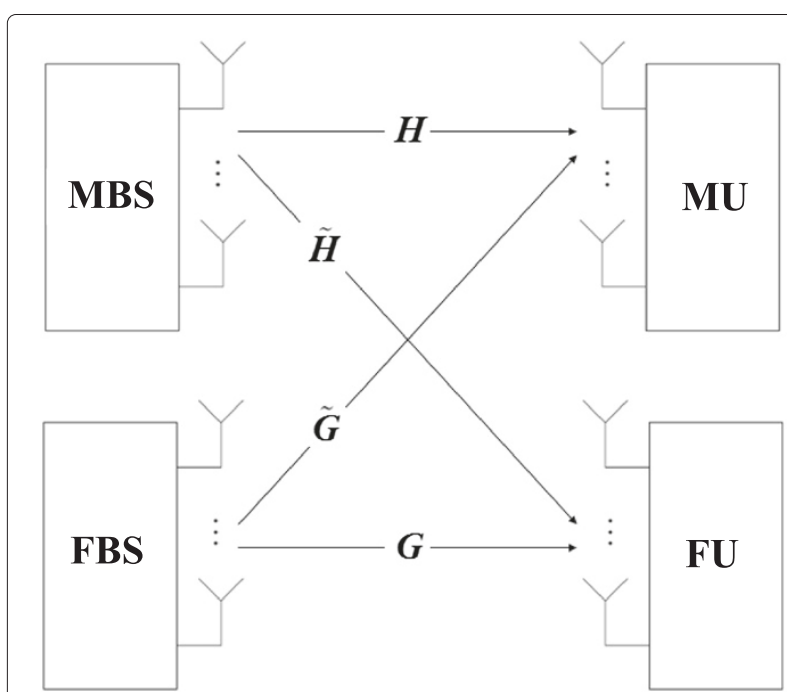

Figure 2 Channel model for downlink transmissions in a two-tier HetNet.

channel state information (CSI) reporting from the users or in case of time-division duplex (TDD) by measuring the uplink channel and considering channel reciprocity.

However, in a practical system with frequency division duplex (FDD), full channel knowledge is very difficult to realize. Therefore, we present how the proposed scheme could be adapted to more realistic assumptions with limited CSI feedback, where the CSI reports are quantized. This feedback includes a channel quality indicator (CQI) and optionally a precoding matrix indicator (PMI). Furthermore, in a realistic system, the CSI feedback is reported for a given TTI but used for a later downlink transmission. Thus, we apply a delay of $T$ TTIs from the time the CSI is received by a BS until the time it is used for scheduling the downlink resources. The simulation results presented in Section 4 consider both cases of full channel knowledge and limited CSI feedback.

\section{Transmission schemes}

Interference management is crucial for HetNets to avoid low signal quality and eventually low system throughput. To this end, a new transmission scheme is developed and discussed in Section 3.3. Before that, two other solutions, based on existing schemes, are presented in Sections 3.1 and 3.2, respectively, in order to be used as reference solutions. The first reference scheme is non-cognitive and is based on enhanced inter-cell interference coordination (eICIC), as defined for LTE-Advanced [22]. The second reference scheme relies on the CR technique of [17], where the MBS is a legacy node and the FBSs are cognitive nodes. These schemes are chosen as references due to their suitability to HetNets and the similarity of their interference management requirements to the proposed solution.

\subsection{Enhanced inter-cell interference coordination}

Enhanced inter-cell interference coordination has been specified for LTE-Advanced as a means to manage intercell interference and allows for a frequency reuse factor of 1 . The concept of 'almost-blank subframe' (ABS) has been introduced, where during such ABS subframes, no data transmission takes place and only the control channels are active with very low power. eICIC uses almostblank subframes to provide smart resource allocation among interfering cells and improve inter-cell load balancing in heterogeneous networks. The BSs coordinate their transmissions over the common spectrum by applying non-overlapping ABS configurations. An example of ABS configuration is shown in Figure 3. When the MBS configures an ABS subframe, then the FBS transmits normally and the FUs receive data without any interference from the MBS. Similarly, when a FBS configures an ABS subframe, the MUs can receive data from the MBS. The ABS configurations can be negotiated between the BSs via the connecting fiber link (e.g., for LTE-Advanced, the X2 interface is defined for this purpose).

In the eICIC scheme, cross-tier intercell interference between MBS and FBS is handled via time-division duplexing and thus no precoding is required. The received signals in this case read

$$
\begin{aligned}
& \text { Macro-user : } \boldsymbol{y}_{p}=P_{p} \boldsymbol{H} x_{p}+\boldsymbol{z}_{p} \\
& \text { Femto-user }: \boldsymbol{y}_{s}=P_{s} \boldsymbol{G} x_{s}+\boldsymbol{z}_{s}
\end{aligned}
$$

and the signal-to-noise ratio (SNR) is calculated as

$$
\begin{aligned}
& \operatorname{SNR}^{t, f}(p)=\frac{P_{p}^{2}\|\mathbf{H}\|^{2}}{\sigma_{n}^{2}} \\
& \operatorname{SNR}^{t, f}(s)=\frac{P_{s}^{2}\|\mathbf{G}\|^{2}}{\sigma_{n}^{2}},
\end{aligned}
$$

where $\sigma_{n}^{2}$ is the noise variance.

As the focus is on optimizing spectral efficiency, each BS schedules the user with the highest SNR. So, for every non-ABS TTI $t$ and every $\operatorname{PRB} f$, the MBS schedules user $p_{\max }$ with

$$
\operatorname{SNR}^{t, f}\left(p_{\max }\right)=\max _{p=1, \ldots P} \operatorname{SNR}^{t, f}(p)
$$

and the FBS schedules user $s_{\max }$ with

$$
\operatorname{SNR}^{t, f}\left(s_{\max }\right)=\max _{s=1, \ldots S} \operatorname{SNR}^{t, f}(s) \text {. }
$$

The main disadvantage of this scheme lies in the fact that no concurrent spectrum access is possible for the interfering BSs. Each BS is allowed to access the spectrum 


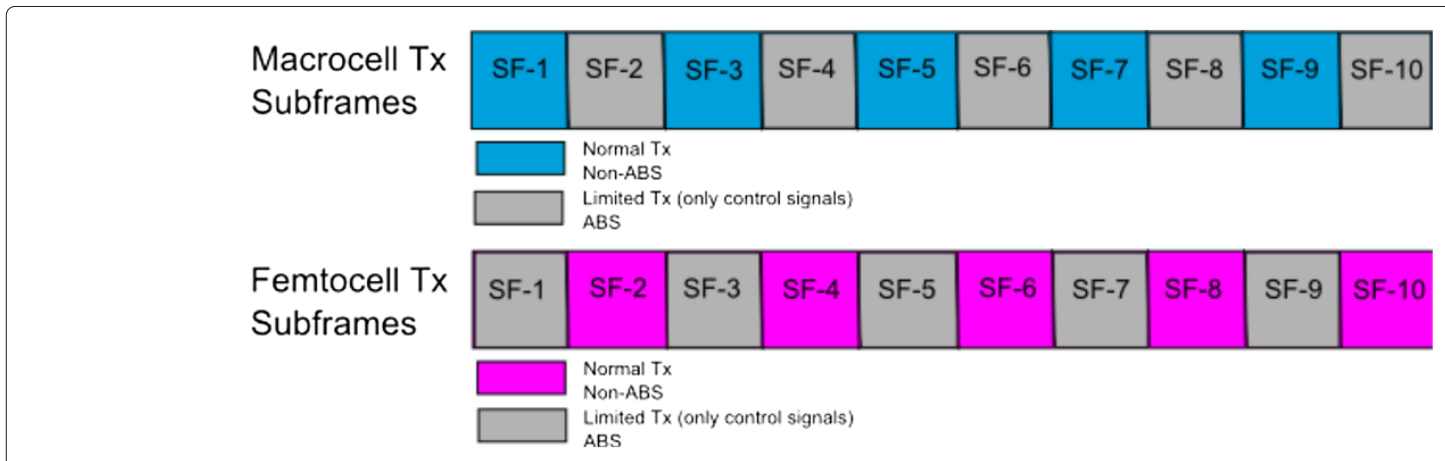

Figure 3 eICIC ABS configuration example for HetNets.

only during non-ABS subframes, which in turn limits the achieved spectral efficiency. eICIC could be combined and enhanced with other techniques. For example, the available spectrum could be divided in a common part which the BSs share and a dedicated part only for FBS transmissions. Nevertheless, in this work, we consider as reference the standard eICIC technique with no enhancements.

\subsection{Femto-cognition with legacy macrocell}

The CR reference scheme is an underlay one and is based on the scheme proposed in [17]. The macrocell is considered as the primary network, which has absolute priority to access the available spectrum. It is a legacy (noncognitive) node and is unaware of the existence of FBSs. On the contrary, the femtocells access the spectrum as secondary networks provided that they do not harm the performance of the primary network significantly. This means that the cross-tier intercell interference from a FBS to the MBS should not exceed a predefined interference threshold $I_{\max }$.

As the primary network is assumed to operate without taking into account the secondary network, the optimal way for the MBS to maximize capacity is to perform a singular value decomposition (SVD) of the downlink channel matrix

$$
\boldsymbol{H}=\boldsymbol{U}_{\boldsymbol{h}} \boldsymbol{\Lambda}_{\boldsymbol{h}} \boldsymbol{V}_{\boldsymbol{h}}^{\boldsymbol{H}}=\sum_{i=1}^{M} \lambda_{h}(i) \boldsymbol{u}_{h}(i) \boldsymbol{v}_{h}(i)^{H}
$$

and then apply a beamforming vector $\boldsymbol{w}_{p}$, such that $\boldsymbol{w}_{p}=$ $\boldsymbol{v}_{\boldsymbol{h}}(1)$, where $\boldsymbol{U}_{\boldsymbol{h}}, \boldsymbol{V}_{\boldsymbol{h}}$ are unitary matrices and $\boldsymbol{\Lambda}_{\boldsymbol{h}}$ is a diagonal matrix which has the singular values ordered over the diagonal. The MBS makes use of the strongest eigenchannel with a single data stream being transmitted.

Similar to [17], the FBS computes the SVD of $\tilde{\boldsymbol{G}}$ as

$$
\tilde{\boldsymbol{G}}=\boldsymbol{U}_{\tilde{g}} \boldsymbol{\Lambda}_{\tilde{g}} \boldsymbol{V}_{\tilde{g}}^{H}=\sum_{i=1}^{M} \lambda_{\tilde{g}}(i) \boldsymbol{u}_{\tilde{g}}(i) \boldsymbol{v}_{\tilde{g}}(i)^{H}
$$

and aims at minimizing the interference to the MU. As the signals might not be orthogonal, the authors suggest that the FU signal should be precoded according to the weakest eigenmode of the interfering channel $\tilde{\boldsymbol{G}}$. Such a precoding ensures that the FU signal transmitted through the crosschannel $\tilde{\boldsymbol{G}}$ will be the weakest possible, and hence, the resulting cross-tier interference will be minimized. Therefore, the precoding vector is set to $\boldsymbol{w}_{s}=\boldsymbol{v}_{\tilde{g}}(M)^{H}$. Then, the signal model reads

$$
\begin{aligned}
& \boldsymbol{y}_{p}=P_{p} \lambda_{h}(1) \boldsymbol{u}_{h}(1) x_{p}+P_{s} \lambda_{\tilde{g}}(M) \boldsymbol{u}_{\tilde{g}}(M) x_{s}+\mathbf{z}_{p} \\
& \boldsymbol{y}_{s}=P_{s} \boldsymbol{G} \boldsymbol{v}_{\tilde{g}}(M) x_{s}+P_{p} \tilde{\boldsymbol{H}} \boldsymbol{v}_{h}(1) x_{p}+\mathbf{z}_{s}
\end{aligned}
$$

Assuming that the users employ maximal ratio combining (MRC) receiver filters, the signal-to-interference and noise ratio (SINR) of the $p$ th MU can be written as

$$
\operatorname{SINR}(p)=\frac{P_{p}^{2} \lambda_{h}^{2}(1)}{P_{s}^{2} \lambda_{\tilde{g}}^{2}(M)\left\|\left\langle\boldsymbol{u}_{h}(1), \boldsymbol{u}_{\tilde{g}}(M)\right\rangle\right\|^{2}+N_{0}}
$$

Similar to the eICIC scheme and in order to maximize the macrocell capacity, the MBS schedules the user with the highest SNR among all MUs. For every TTI $t$ and every $\operatorname{PRB} f$, the MBS schedules the MU $p_{\max }$ with

$$
\operatorname{SNR}^{t, f}\left(p_{\max }\right)=\max _{p=1, \ldots P} \operatorname{SNR}^{t, f}(p),
$$

where the SNR is calculated as

$$
\operatorname{SNR}^{t, f}(p)=\frac{P_{p}^{2}\|\boldsymbol{H}\|^{2}}{\sigma_{n}^{2}} .
$$

On the other hand, the FBS selects the FU $s_{\min }$ that minimizes the interference caused by the $\mathrm{MU} p_{\max }$

$$
I\left(s_{\min }\right)=\min _{s=1, \ldots S} I(s),
$$

where $I(s)$ is defined as

$$
I(s)=\lambda_{\tilde{g}}^{2}(M)\left\|\left\langle\boldsymbol{u}_{h}(1), \boldsymbol{u}_{\tilde{g}}(M)\right\rangle\right\|^{2} .
$$


If the interference constraint $I\left(s_{\min }\right) \leq I_{\max }$ is satisfied, then the FBS schedules the FU $s_{\min }$. Otherwise, the FBS does not schedule any FU.

Note that according to this scheme, the primary user selection maximizes the primary network rate, whereas the secondary user selection ensures only the minimum interference to the primary network. Since the channels are independent, the rate of the secondary users becomes eventually a random variable. This means that multi-user diversity and frequency selectivity are used only to limit the interference and not to improve the overall spectral efficiency. Consequently, the primary network is given absolute priority.

\subsection{Joint precoding based on cognition with full channel knowledge}

In the proposed scheme, the two main weaknesses of the reference and other existing solutions are addressed, namely the lack of simultaneous spectrum access and the user prioritization. We consider full system cognition and multi-cell cooperation, where all BSs are cognitive nodes and the available spectrum is accessed by both macroand femtocells simultaneously. The BSs are aware of the deployment and exchange TTI-based information over the available fiber links, taking full advantage of the system architecture. Specifically, the scheduling and precoding decisions are taken centrally but in a cooperative way and are then distributed via the fiber links to both MBS and FBS for transmission.

The signal model for each pair of macro- and femtoreceivers is given in (1) and (2), but to simplify the equations, we use a more compact notation as follows:

$$
\begin{aligned}
\boldsymbol{H}_{p} & =[\boldsymbol{H}, \tilde{\boldsymbol{G}}], \\
\boldsymbol{H}_{s} & =[\tilde{\boldsymbol{H}}, \boldsymbol{G}], \\
\boldsymbol{W} & =\left[\boldsymbol{w}_{p}, \boldsymbol{w}_{s}\right] \\
\boldsymbol{x} & =\left[x_{p}, x_{s}\right] \\
\boldsymbol{A}_{p} & =\boldsymbol{H}_{p} \boldsymbol{W}, \\
& \equiv\left[\boldsymbol{a}_{p}, \tilde{\boldsymbol{a}}_{p}\right], \\
\boldsymbol{A}_{s} & =\boldsymbol{H}_{s} \boldsymbol{W}, \\
& \equiv\left[\tilde{\boldsymbol{a}}_{s}, \boldsymbol{a}_{s}\right], \\
\tilde{\boldsymbol{n}}_{p} & =\tilde{\boldsymbol{a}}_{p} \boldsymbol{x}_{s}+\boldsymbol{n}_{p} \\
\tilde{\boldsymbol{n}}_{s} & =\tilde{\boldsymbol{a}}_{s} \boldsymbol{x}_{p}+\boldsymbol{n}_{s}
\end{aligned}
$$

Hence, $\boldsymbol{A}_{u}$ with $u \in\{p, s\}$ denotes the aggregate channel matrix in the transmission of signal $\boldsymbol{x}$ to user $u$ and $\tilde{\boldsymbol{n}}_{u}$ denotes the aggregate noise and interference.

In order to mitigate the cross-tier intercell interference, zero-forcing (ZF) precoding at the transmitter sides is applied. The goal is to introduce pre-distortion to the channels already at the BS side using a precoding matrix
$\boldsymbol{W}$, such that the overall MIMO channel $\boldsymbol{A}_{u}$ ideally degenerates to a number of parallel non-interfering channels. The CSI for each user is used to generate an aggregate MIMO channel matrix that is well conditioned for spatial pre-equalization by means of a linear zero-forcing precoder. Provided that the transmitter has perfect channel knowledge, this can be achieved perfectly by using a linear zero-forcing-based precoder.

Assuming that the $p$ th $\mathrm{MU}$ and $s$ th FU are scheduled, the joint ZF precoding matrix $W$ is computed as

$$
\boldsymbol{W}=\boldsymbol{C}_{p, s}^{H}\left(\boldsymbol{C}_{p, s} \boldsymbol{C}_{p, s}^{H}\right)^{-1},
$$

where we define

$$
\boldsymbol{C}_{p, s}=\left[\boldsymbol{h}_{n}^{(p)}, \boldsymbol{h}_{n}^{(s)}\right]
$$

and $\boldsymbol{h}_{n}^{(p)}=\left[\boldsymbol{H}^{(p)}\right]_{:, n}$ for the $p$ th MU, $\boldsymbol{h}_{n}^{(s)}=\left[\boldsymbol{G}^{(s)}\right]_{:, n}$ for the $s$ th FU and $n$ denotes the antenna with the highest channel gain for each user. Note that the joint transmit filter $W$ is designed by forcing the interference from a BS to the user with the highest channel gain to be equal to zero. Instead of performing ZF precoding such that all receive antennas of each user receive only the desired signal, each BS points the transmission to one of the receive antennas of the served user, the antenna with the highest channel gain. At the other receive antennas the user still receives some residual interference, which can however be reduced by the receive filter. The reason for this approach is that in case of ideal CSI at the BS, there is no coherence gain to be obtained at the user side. Thus, it is preferable to focus on the antenna with the highest channel gain to improve the achieved throughput.

Regarding signal reception, we suggest two alternatives based on the user capabilities. In the first alternative, the users are non-cognitive; thus, they are unaware of the precoding applied. In the second alternative, the users are cognitive and can estimate the aggregate channel $\tilde{\boldsymbol{a}}_{u}$ on which interference is received, based on embedded user-specific reference symbols. Such user-specific channel state information reference symbols (CSI-RS) have already been defined in recent releases of LTE-Advanced and could be used for this purpose.

More specifically, if the user $u$ is non-cognitive, then a MRC filter may be employed at the receiver sides. The receive filter in this case is $\boldsymbol{\beta}_{u}=\boldsymbol{a}_{u}$, and the SINR is given by

$$
\operatorname{SINR}_{M R C, u}=\frac{P_{p}^{2}\left\|\boldsymbol{a}_{u}\right\|^{2}}{P_{s}^{2} \frac{\left\|\left\langle\boldsymbol{a}_{u}^{H}, \tilde{\boldsymbol{a}}_{u}\right\rangle\right\|^{2}}{\left\|\boldsymbol{a}_{u}\right\|^{2}}+\sigma_{n}^{2}} .
$$

On the other hand, if the user $u$ is cognitive, it can perform an additional step of equalizing the residual 
interference with a linear minimum mean square error (LMMSE) spatial equalizer. The receive filter then reads

$$
\boldsymbol{\beta}_{u}=\Psi_{\tilde{\boldsymbol{n}}_{u} \tilde{\boldsymbol{n}}_{u}}^{-1} \boldsymbol{a}_{u}
$$

where

$$
\begin{aligned}
\Psi_{\tilde{\boldsymbol{n}}_{u} \tilde{\boldsymbol{n}}_{u}} & =\varepsilon\left\{\tilde{\boldsymbol{n}}_{u} \tilde{\boldsymbol{n}}_{u}^{H}\right\} \\
& =P_{s}^{2} \tilde{\boldsymbol{a}}_{u} \tilde{\boldsymbol{a}}_{u}^{H}+\sigma_{n}^{2} \boldsymbol{I}_{N}
\end{aligned}
$$

and $\sigma_{n}^{2}$ is the noise variance. The SINR can be calculated as

$$
\mathrm{SINR}_{\mathrm{LMMSE}, u}=P_{p}^{2} \boldsymbol{\beta}_{u}^{H} \boldsymbol{a}_{u} .
$$

The user selection is performed in a centralized fashion aiming at maximizing the sum throughput of the MBS and FBS. Thus, for every TTI $t$ and every $\operatorname{PRB} f$, the MU $p_{\max }$ and FU $s_{\max }$ are scheduled, where now

$$
\left(p_{\max }, s_{\max }\right)=\arg \max _{p, s}\left(\mathrm{SINR}_{\text {filter }, p}+\mathrm{SINR}_{\text {filter }, s}\right)
$$

and filter denotes either LMMSE or MRC. Note that the user selection criterion applied in the proposed scheme could be easily modified to follow other scheduling strategies (e.g., proportional fair scheduling).

\subsection{Joint precoding based on cognition with limited CSI feedback}

As perfect channel knowledge is not available in a real mobile communication system, we briefly discuss how the proposed scheme can be formulated based on more realistic assumptions. In this case, the transmit filter computation cannot be based on the actual channel vectors, but instead on the user CSI reports. The users, hence, inform the BS about the current state of their channels by sending quantized and delayed CSI reports. These CSI reports include a channel quality indicator (CQI) and a precoding matrix indicator (PMI), while the PMI reports belong to predefined codebooks.

Given some codebook $\mathcal{C}=\left\{c_{1}, \ldots, c_{|\mathcal{C}|}\right\}$ of unit normal vectors, each user $u$ selects the representative of the channel vectors connecting the $M$ transmit antennas of the BS to the $n$th receive antenna according to

$$
\hat{\boldsymbol{h}}_{n}^{(u)}=\arg \max _{\boldsymbol{c}_{\nu} \in \mathbf{C}}\left\{\left|\boldsymbol{c}_{\nu}^{H} \boldsymbol{h}_{n}^{(u)}\right|\right\},
$$

where $\boldsymbol{h}_{n}^{(u)}=\left[\boldsymbol{H}^{(u)}\right]_{:, n}$ for a MU and $\boldsymbol{h}_{n}^{(u)}=\left[\boldsymbol{G}^{(u)}\right]_{:, n}$ for a FU. The user reports a single PMI value $\mathrm{PMI}_{u}$, which corresponds to the codebook vector that best represents one of its channel vectors.

Assuming that the $p$ th $\mathrm{MU}$ and $s$ th FU are scheduled, the joint ZF precoding matrix $W$ is computed according to the user PMI reports as

$$
\boldsymbol{W}=C_{p, s}^{H}\left(C_{p, s} C_{p, s}^{H}\right)^{-1}
$$

where we define

$$
\boldsymbol{C}_{p, s}=\left[\boldsymbol{c}_{\mathrm{PMI}_{p}}, \boldsymbol{c}_{\mathrm{PMI}_{s}}\right]
$$

Due to the imperfect channel knowledge, some residual interference remains. Therefore, the receive filter selection is more important in this case compared to the case of ideal CSI. The rest of the scheme remains the same.

\section{Simulation results and performance evaluations}

Extensive simulations have been performed to evaluate the transmission schemes presented in the previous section. The two variations of the proposed scheme (joint precoding based on cognition (JPC)) are compared to the two reference solutions (eICIC, femto-cognition with legacy macrocell (FCLM)), as well as the case where no interference management (No IM) is applied. We consider a single macrocell serving $P=100$ users and $F=4$ femtocells serving $S=25$ users each. The bandwidth of each carrier is set to $B=5 \mathrm{MHz}$, consisting of $25 \mathrm{PRBs}$ of 180 $\mathrm{KHz}$ each. The number of antennas is set to $M=N_{\mathrm{MBS}}=$ $N_{\text {FBS }}=2$. The TTI is set to $1 \mathrm{~ms}$ and the overall simulation time is 100,000 TTIs. The downlink channels are considered multi-path and time invariant over a TTI, but change independently for every subsequent TTI. In case of FCLM scheme, full channel knowledge is assumed. For eICIC and JPC schemes, we consider both full channel knowledge and limited CSI reporting. In the latter, each user reports a CQI value of 6 bits per PRB and TTI. A PMI value of 3 bits is additionally reported in case of JPC scheme, where Grassmannian codebooks are used. The delay of all reports is set to $T=4 \mathrm{~ms}$. Finally, for the eICIC scheme, we set every even TTI to be an ABS for the MBS and every odd TTI to be an ABS for the FBSs, as shown in Figure 3. We have also simulated other ABS patterns, which are not presented here due to space limitations and because they showed similar performance. Table 1 summarizes the simulation parameters.

In order to ensure fairness among the users and focus on the transmission schemes, users are assumed to be stationary with common and constant pathloss to the serving BS. We consider no power control, i.e., each BS applies constant power to all users served, with $P_{p}^{2}=43 \mathrm{dBm}$ and $P_{s}^{2}=23 \mathrm{dBm}[25]$.

In Figures 4 and 5, the cumulative distribution function (CDF) of the effective SINR is shown for the macrocell and femtocell users, respectively. As expected, the highest SINR for both MUs and SUs is observed for the eICIC scheme, since only one of the BS transmits at aiven TTI; thus, no inter-cell interference occurs. Moreover, the cognitive reference solution (FCLM) provides higher effective SINR for the MUs compared to the proposed scheme (JPC). This can be explained by the fact that each FBS allocates the resources and aligns its transmissions so as 
Table 1 Simulation parameters

\begin{tabular}{|c|c|}
\hline Parameter & Value \\
\hline Number of macrocells & 1 \\
\hline Number of femtocells & 4 \\
\hline Number of macrocell users & 100/cell \\
\hline Number of femtocell users & 25/cell \\
\hline \multicolumn{2}{|l|}{ System bandwidth } \\
\hline Macrocell & $20 \mathrm{MHz}$ \\
\hline Femtocell & $5 \mathrm{MHz}$ \\
\hline Physical resource block & $180 \mathrm{KHz}$ \\
\hline Distance-dependent pathloss & $128.1+37.6 \log ($ distance $)$ \\
\hline Shadowing & None \\
\hline Tा। & $1 \mathrm{~ms}$ \\
\hline \multicolumn{2}{|l|}{ Total BS transmission power } \\
\hline macrocell & $43 \mathrm{dBm}$ \\
\hline Femtocell & $23 \mathrm{dBm}$ \\
\hline Number of BS Tx antennas & 2 \\
\hline Number of UE Rx antennas & 2 \\
\hline Bits per CQI report & 6 \\
\hline Bits per PMI report & 3 \\
\hline Codebook type & Grassmannian \\
\hline CSI report delay & $4 \pi / s$ \\
\hline Frequency reuse factor & 1 \\
\hline Frequency domain scheduler & Max rate \\
\hline Simulation time & 100,000 TT/s \\
\hline
\end{tabular}

to minimize the interference to the MUs. However, this causes the SINR of the SUs to suffer significantly. From Figure 5, it is obvious that the received signal quality for the FUs in case of FCLM is much lower than that for JPC.

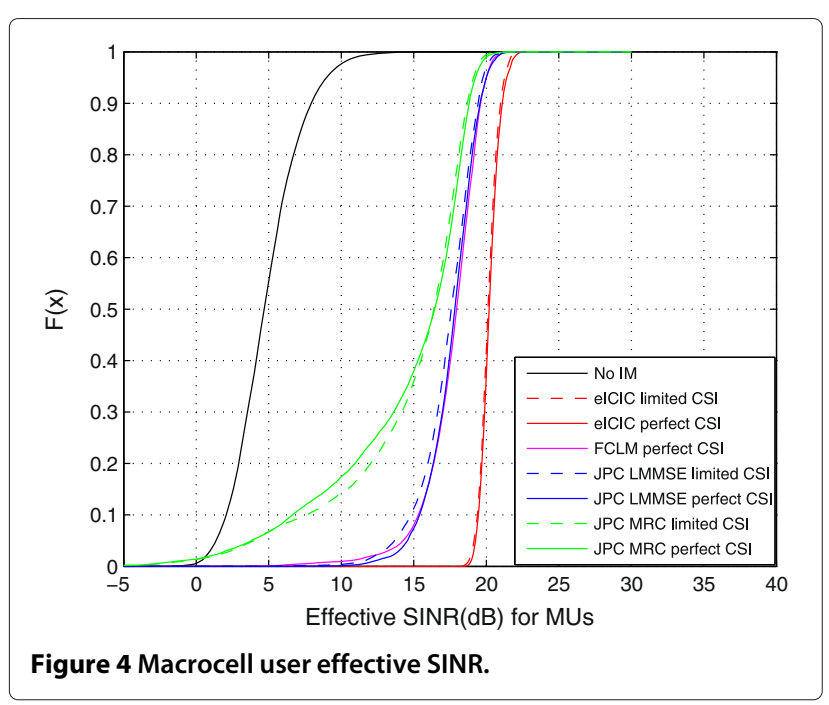

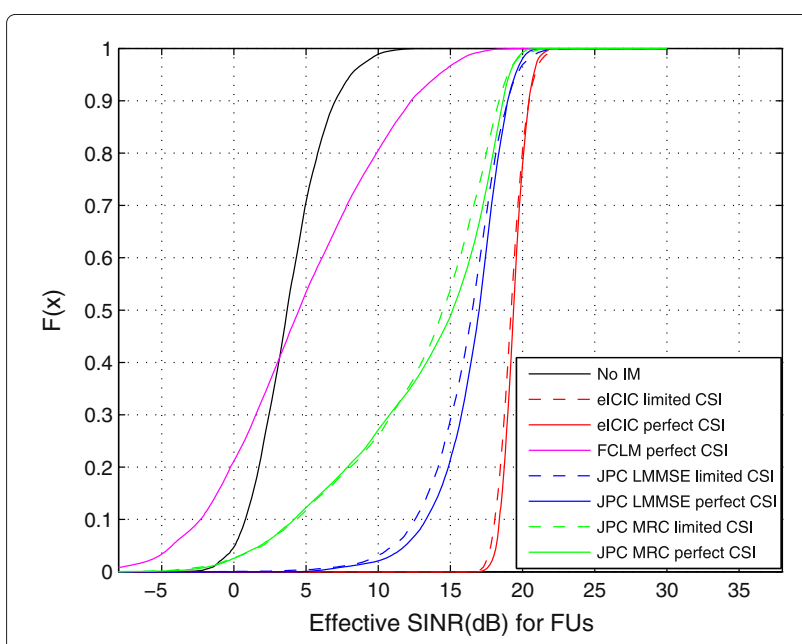

Figure 5 Femtocell user effective SINR.

Figure 6 illustrates the CDF of the aggregate throughput achieved by the system for the investigated schemes. Although the eICIC scheme provides the highest effective SINR, it achieves the lowest aggregate throughput, as the spectral efficiency is poor due to the time-division duplexing applied. On the other hand, the cognitive reference solution (i.e., FCLM) provides clear gains of around 22\% over the non-cognitive eICIC. Yet, one can observe in Figure 7 that the femtocell rates are rather low $(32 \%$ lower than eICIC). This can be attributed to the downprioritization of the FUs and the fact that the femtocell resource allocation strategy focuses on limiting the interference to the MUs and not on the optimization of the overall spectral efficiency.

The proposed JPC scheme outperforms both reference solutions and achieves large gains over them. JPC

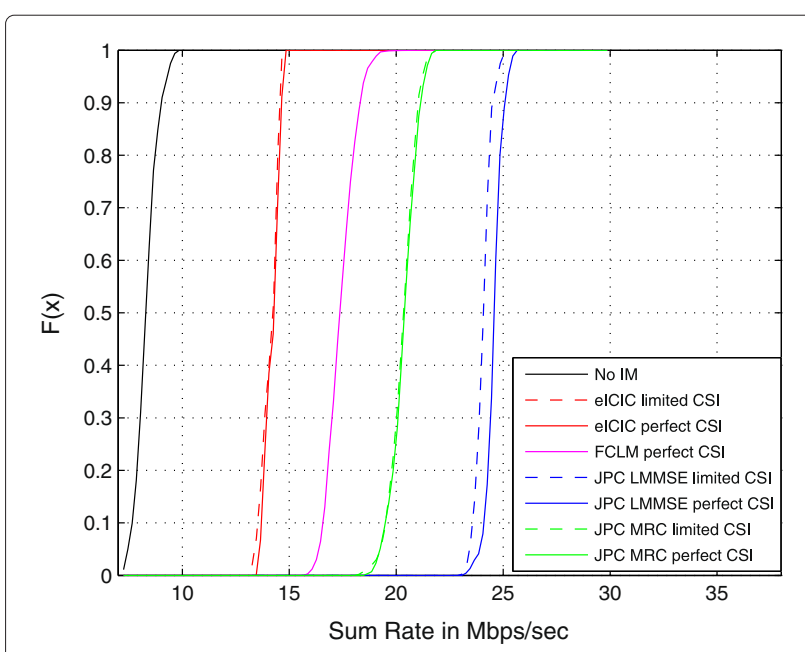

Figure 6 Aggregate rate of the two-tier HetNet. 


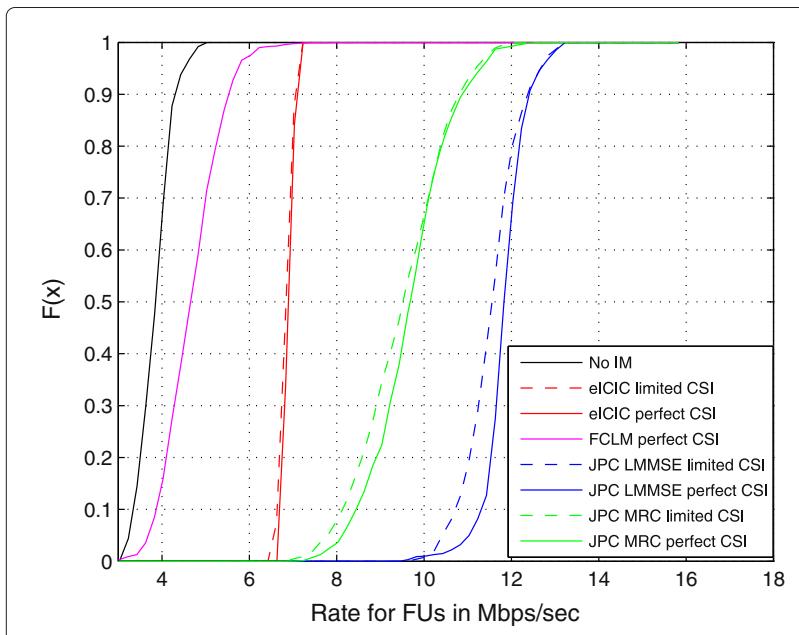

Figure 7 Femtocell rate of the two-tier HetNet.

achieves up to $41 \%$ higher throughput compared to FCLM and up to $72 \%$ compared to eICIC. The joint resource allocation and BS cooperation allow for performing efficient precoding, while scheduling users with high channel gains. Moreover, the use of CSI-RS in conjunction with a LMMSE receiver boosts the throughput even more, compared to the simple MRC filter. Figure 8 depicts the throughput achieved by the MUs for each transmission scheme. JPC achieves significant rate improvement for both MUs and FUs, as well as for the aggregate system throughput. Note that these performance gains come at the expense of extra CSI information and higher complexity for BS cooperation. However, this is possible given the network architecture (HetNet with RRHs).

Comparing the performance of JPC for ideal and limited CSI, small losses are observed due to the imperfect $\mathrm{ZF}$ precoding. The losses are smaller when a LMMSE

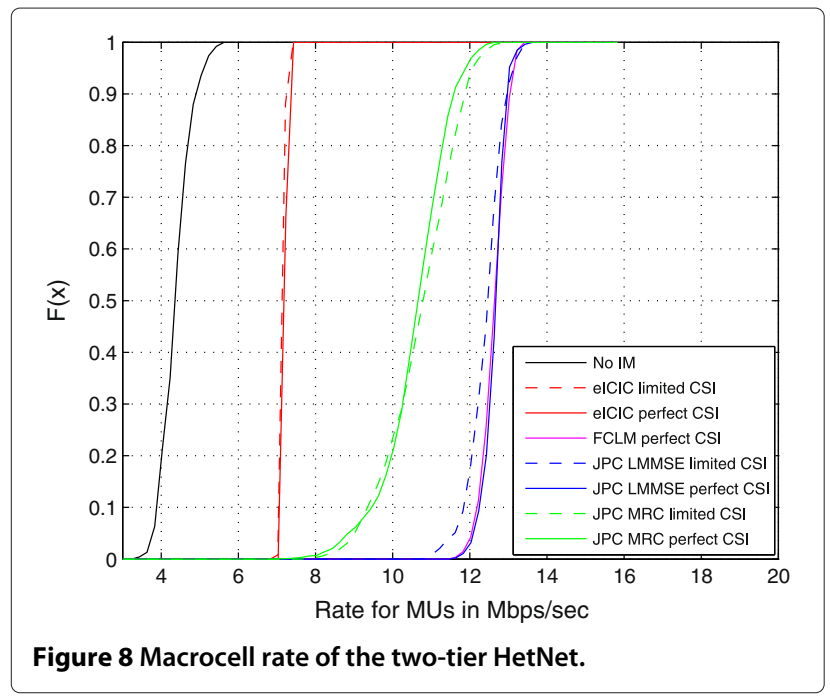

receive filter (2\%) is applied compared to a MRC filter (5\%). The receive filter selection is more important in case of limited CSI. This is expected since the LMMSE filter cancels out the remaining interference at the receiving side. The simulation results also show that the way the ZF precoding matrix is computed is efficient even for imperfect CSI, especially in the case of a LMMSE receive filter.

Finally, Table 2 summarizes the comparison of the different alternatives. From this table, it is clear that interference management is crucial and that the proposed scheme leads to higher aggregate throughput of the overall system due to more efficient interference management. The throughput improvement may perfectly justify the slightly higher (but manageable) CSI requirements of the proposed method.

\section{Conclusions}

HetNet technology combined with efficient interference management can provide increased network capacity to the mobile subscribers and the service providers. In this article, we have discussed different interference management techniques for HetNets. A novel scheme based on full system cognition and multi-cell cooperation for a two-tier cellular system is developed and proposed. This scheme applies joint precoding in conjunction with resource allocation and turns out to outperform the solution that is currently specified for LTE-Advanced. We have shown how the proposed scheme can be adapted to realistic network assumptions and limited CSI reporting.

Table 2 Comparison of interference management schemes

\begin{tabular}{lccc}
\hline IM scheme & $\begin{array}{c}\text { Macrocell } \\
\text { throughput } \\
\text { (Mbps) }\end{array}$ & $\begin{array}{c}\text { Femtocell } \\
\text { throughput } \\
\text { (Mbps) }\end{array}$ & $\begin{array}{c}\text { Aggregate } \\
\text { throughput } \\
\text { (Mbps) }\end{array}$ \\
\hline No IM & 4.47 & 3.94 & 8.41 \\
elCIC & 7.26 & 6.94 & 14.20 \\
limited CSI & 7.29 & 6.99 & 14.28 \\
elCIC & & & \\
perfect CSI & 12.73 & 4.77 & 17.50 \\
FCLM & & & 19.42 \\
perfect CSI & 10.30 & 9.12 & 20.45 \\
JPC MRC & & & \\
limited CSI & 10.68 & 9.77 & 24.15 \\
JPC MRC & & 11.64 & \\
perfect CSI & 12.51 & & \\
JPC LMMSE & & 11.94 & \\
limited CSI & 12.73 & & \\
JPC LMMSE & & & \\
perfect CSI & & & \\
\hline
\end{tabular}


We have also investigated how the receive filter selection affects the achieved performance. Furthermore, we have shown that applying cognition to both the macrocell and femtocell base stations leads to significant gains for the femtocells compared to a traditional cognitive radio system where femto-users are down-prioritized over macrocell users. This is crucial, as femtocells can be considered to be a viable network solution only if they can ensure high data rate for their subscribers.

\section{Competing interests}

The authors declare that they have no competing interests.

\section{Authors' contributions}

IT and KB conceived and designed the proposed JPC transmission scheme. IT designed and performed the simulations, drafted the manuscript and KB revised the manuscript. All authors read and approved the final manuscript.

\section{Acknowledgements}

This work was supported in part by the University of Patras and by EU and national funds via the National Strategic Reference Framework, Research Funding Program THALES.

Received: 11 February 2014 Accepted: 27 July 2014

Published: 4 September 2014

\section{References}

1. SRangan, Femto-macro cellular interference control with subband scheduling and interference cancelation, in IEEE GLOBECOM Workshops (GCWkshps) (Miami, FL, 6-10 Dec 2010), pp. 695-700

2. N Saquib, E Hossain, LB Le, DI Kim, Interference management in OFDMA femtocell networks: issues and approaches. IEEE Wireless Commun. 19(3), 86-95 (2012)

3. X Tao, Z Zhao, R Li, J Palicot, H Zhang, Downlink interference minimization in cooperative cognitive LTE-femtocell networks. EURASIP J. Wireless Commun. Netw. 2013(194) (2013)

4. D Gesbert, S Hanly, H Huang, S Shamai Shitz, O Simeone, W Yu, Multi-cell MIMO cooperative networks: a new look at interference. IEEE J. Select. Areas Commun. 28(9), 1380-1408 (2010)

5. $\quad \mathrm{Y}$ Wu, D Zhang, $\mathrm{H}$ Jiang, $Y$ Wu, A novel spectrum arrangement scheme for femto cell deployment in LTE macro cells, in IEEE 20th International Symposium on Personal, Indoor and Mobile Radio Communications (Tokyo, Japan, 13-16 Sept 2009), pp. 6-11

6. H Li, XXu, D Hu, X Qu, X Tao, P Zhang, Graph method based clustering strategy for femtocell interference management and spectrum efficiency improvement, in 6th International Conference on Wireless Communications Networking and Mobile Computing (WiCOM) (Chengdu, 23-25 Sept 2010), pp. 1-5

7. H Widiarti, S-Y Pyun, D-H Cho, Interference mitigation based on femtocells grouping in low duty operation, in IEEE 72nd Vehicular Technology Conference Fall (VTC 2010-Fall) (Ottawa, ON, Canada, 6-9 Sept 2010), pp. 1-5

8. ME Sahin, I Guvenc, M-R Jeong, H Arslan, Handling CCl and ICl in OFDMA femtocell networks through frequency scheduling. IEEE Trans. Consum. Electron. 55(4), 1936-1944 (2009)

9. T-H Kim, T-J Lee, Throughput enhancement of macro and femto networks by frequency reuse and pilot sensing, in IEEE International Performance, Computing and Communications Conference (IPCCC) (Austin, TX, 7-9 Dec 2008), pp. 390-394

10. P Lee, T Lee, J Jeong, J Shin, Interference management in LTE femtocell systems using fractional frequency reuse, in The 12th International Conference on Advanced Communication Technology (ICACT), vol. 2 (Phoenix Park, 7-10 Feb 2010), pp. 1047-1051

11. R-T Juang, P Ting, H-P Lin, D-B Lin, Interference management of femtocell in macro-cellular networks, in Wireless Telecommunications Symposium (WTS) (Tampa, FL, 2010), pp. 1-4
12. S Guruacharya, D Niyato, E Hossain, DI Kim, Hierarchical competition in femtocell-based cellular networks, in IEEE Global Telecommunications Conference (GLOBECOM 2010) (Miami, FL, 6-10 Dec 2010), pp. 1-5

13. MS Jin, SA Chae, DI Kim, Per cluster based opportunistic power control for heterogeneous networks, in IEEE 73rd Vehicular Technology Conference (VTC Spring) (Budapest, 15-18 May 2011), pp. 1-5

14. A Feistel, S Stańczak, D Tomecki, Joint utility-based power control and receive beamforming in decentralized wireless networks. EURASIP J Wirel Comm. 2010, 751893 (2010)

15. S Srinivasa, SA Jafar, Cognitive radios for dynamic spectrum access - the throughput potential of cognitive radio: a theoretical perspective. IEEE Commun. Mag. 45(5), 73-79 (2007)

16. TYücek, H Arslan, A survey of spectrum sensing algorithms for cognitive radio applications. IEEE Commun. Surv. Tutorials. 11(1), 116-130 (2009)

17. C Shen, MP Fitz, Opportunistic spatial orthogonalization and its application in fading cognitive radio networks. IEEE J. Select. Topics Signal Process. 5(1), 182-189 (2011)

18. G Guĺr, S Bayhan, F Alagoĺz, Cognitive femtocell networks: an overlay architecture for localized dynamic spectrum access [dynamic spectrum management]. IEEE Wireless Commun. 17(4), 62-70 (2010)

19. A Adhikary, V Ntranos, G Caire, Cognitive femtocells: breaking the spatial reuse barrier of cellular systems, in Information Theory and Applications Workshop (ITA) (La Jolla, CA, 6-11 Feb 2011), pp. 1-10

20. R Urgaonkar, MJ Neely, Opportunistic cooperation in cognitive femtocell networks. IEEE J. Selected Areas Commun. 30(3), 607-616 (2012)

21. Y Bai, L Chen, Hybrid spectrum arrangement and interference mitigation for coexistence between LTE macrocellular and femtocell networks. EURASIP J. Wireless Comm. Network. 2013(56) (2013)

22. 3rd Generation Partnership Project (3GPP), Technical Specification Group Radio Access Network, Evolved Universal Terrestrial Radio Access (E-UTRA). Overall Description. 3GPP TS 36.300. (3rd Generation Partnership Project (3GPP), New Orleans, 2012)

23. L Brunel, M Plainchault, N Gresset, Dammann A, C Mensing, R Raulefs, Inter-cell interference coordination and synchronization based on location information, in 7th Workshop on Positioning Navigation and Communication (WPNC) (Dresden, 11-12 March 2010), pp. 224-232

24. M Castaneda, MT Ivrlac, JA Nossek, I Viering, A Klein, On downlink intercell interference in a cellular system, in IEEE 18th International Symposium on Personal, Indoor and Mobile Radio Communications (PIMRC) (Athens, 3-7 Sept 2007), pp. 1-5

25. 3rd Generation Partnership Project (3GPP), Technical Specification Group Radio Access Network, Evolved Universal Terrestrial Radio Access (E-UTRA). Base Station (BS) Radio, Transmission and Reception3GPP TS 36.104. (3rd Generation Partnership Project (3GPP), New Orleans, 2012)

doi:10.1186/1687-1499-2014-143

Cite this article as: Tzelatis and Berberidis: Cross-tier interference management schemes in cognitive heterogeneous networks. EURASIP Journal on Wireless Communications and Networking 2014 2014:143.

\section{Submit your manuscript to a SpringerOpen ${ }^{\circ}$ journal and benefit from:}

- Convenient online submission

- Rigorous peer review

- Immediate publication on acceptance

- Open access: articles freely available online

- High visibility within the field

- Retaining the copyright to your article

Submit your next manuscript at $>$ springeropen.com 